\title{
Specialist Gelator for Ionic Liquids
}

Kenji Hanabusa*, Hiroaki Fukui, Masahiro Suzuki, and Hirofusa Shirai

Graduate School of Science and Technology, Shinshu University, Ueda 386-8567, Japan

Supporting I nformation<smiles>CC(C)CCCC(C)CCOC(=O)C[C@@H]1NC(=O)[C@H](Cc2ccccc2)NC1=O</smiles><smiles>CCCCC(CC)COC(=O)C[C@@H]1NC(=O)[C@H](Cc2ccccc2)NC1=O</smiles>

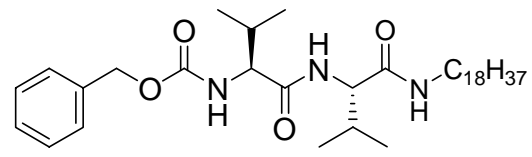

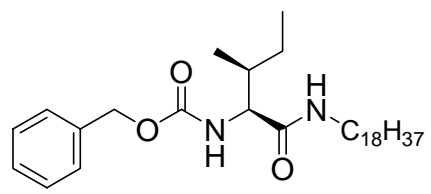

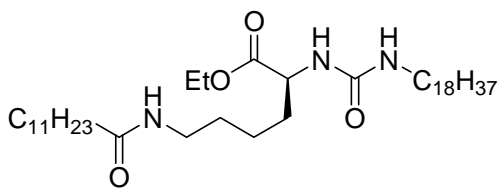

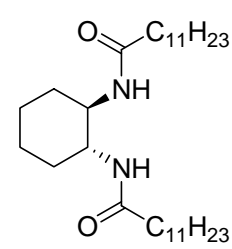

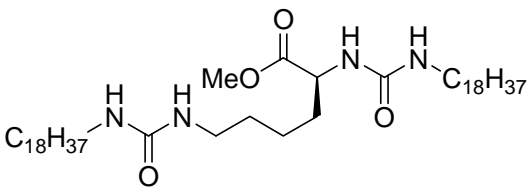

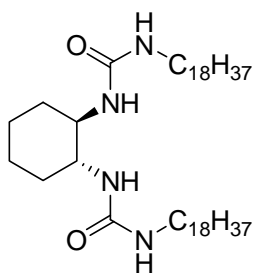<smiles>CCCCCC[C@H](O)CCCCCCCCCCC(=O)O</smiles>

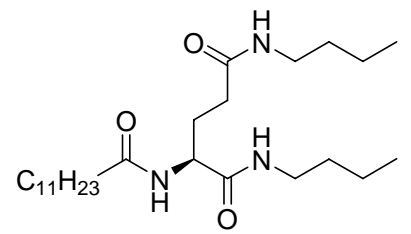

Figure S-1 Structures of twelve kinds of gelators used 


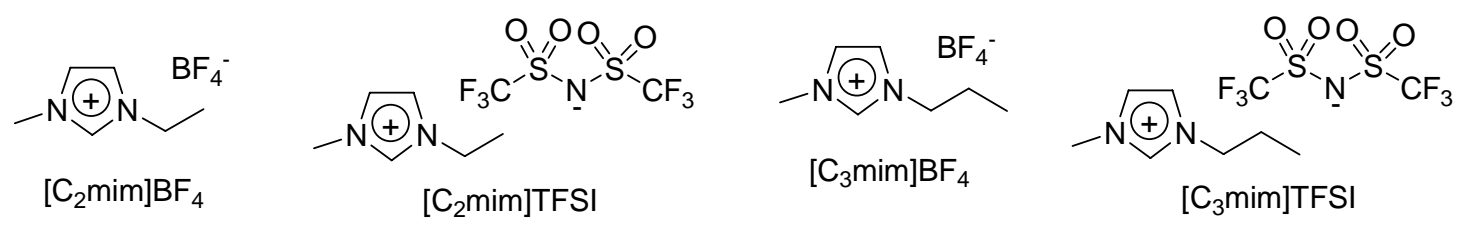

$$
\begin{aligned}
& \text { (1) } \\
& {\left[\mathrm { C } _ { 4 } \mathrm { mim } _ { \mathrm { BF } } \quad \left[\mathrm{C}_{4} \mathrm{mim}_{4} \mathrm{PF}_{6}\right.\right.}
\end{aligned}
$$
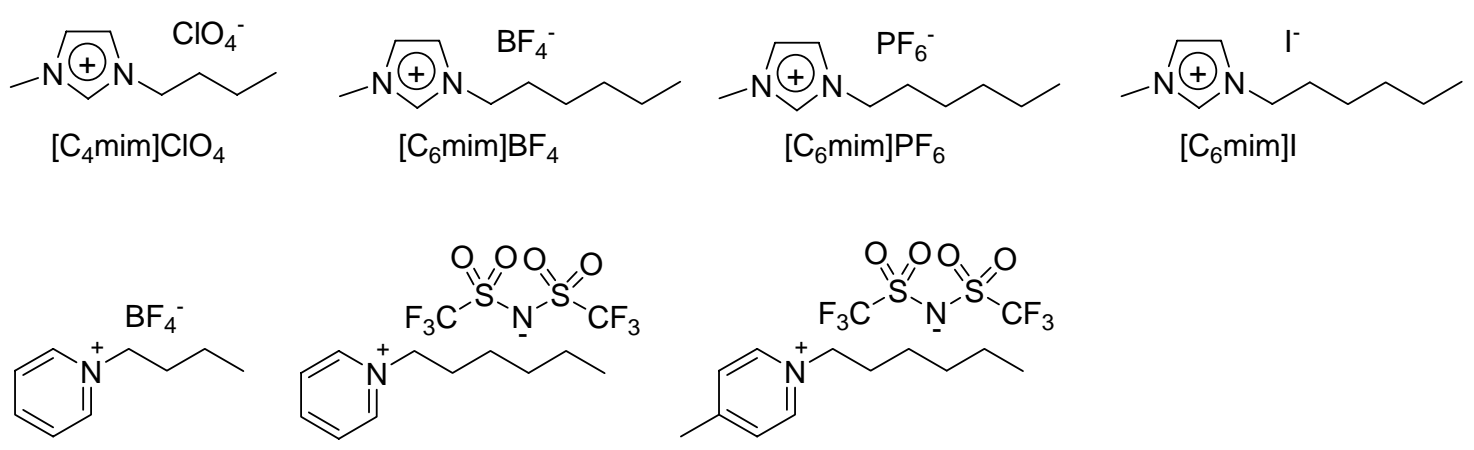

$\left[\mathrm{C}_{4} \mathrm{py}\right] \mathrm{BF}_{4}$

$\left[\mathrm{C}_{6}\right.$ py $]$ TFSI

$\left[\mathrm{C}_{1} \mathrm{C}_{6}\right.$ py]TFSI<smiles>CCCC[N+]1(C)CCCC1</smiles>

$\left[\mathrm{C}_{4}\right.$ pyr $] \mathrm{TFSI}$<smiles>C[N+]1(CCCCN(C(F)(F)F)S(=O)(=O)C(F)(F)F)CCCCC1</smiles>

$\left[\mathrm{C}_{4}\right.$ pip $]$ TFSI

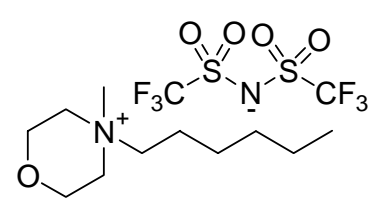

$\left[\mathrm{C}_{6} \mathrm{mor}\right] \mathrm{TFSI}$

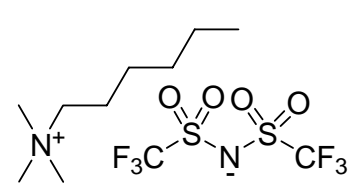

[ammo-1]TFSI

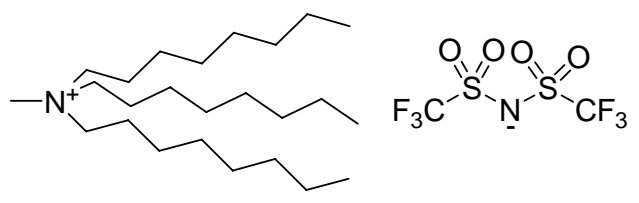

[ammo-2]TFSI

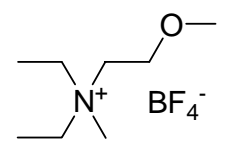

[ammo-3]BF 4

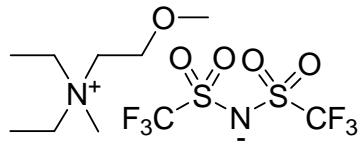

[ammo-3]TFSI

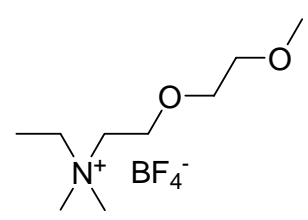

[ammo-4]BF 4

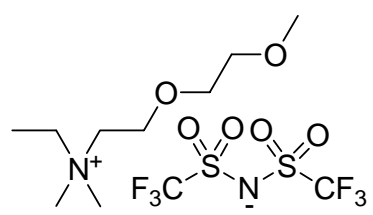

[ammo-4]TFSI

Figure S-2 Structures of ionic liquids 

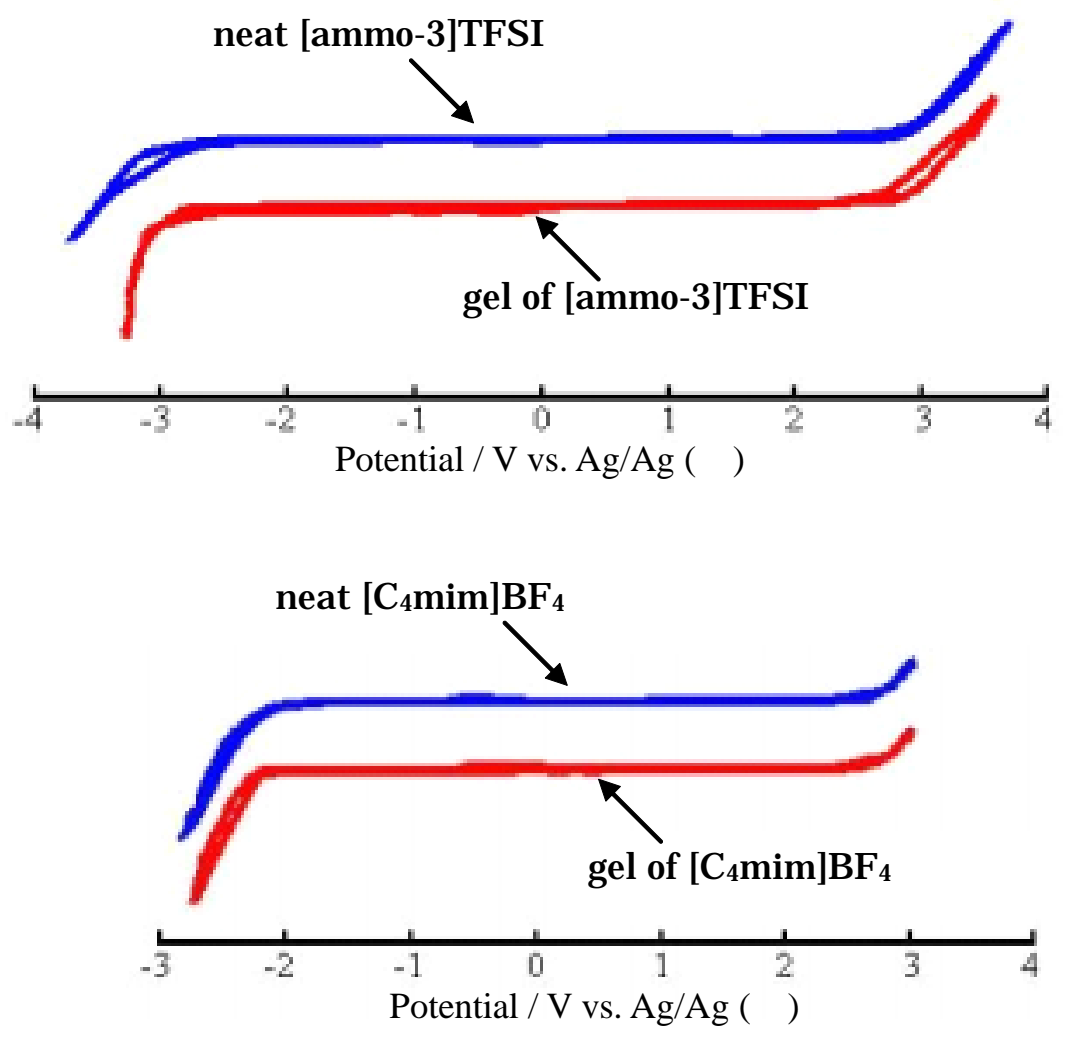

Figure S-3 Cyclic voltammograms of Ag electrode in [ammo-3]TFSI gel (upper) and [C4mim]BF4 gel (lower) formed by gelator 1 . Scan rate $5 \mathrm{mV} \mathrm{s}-1$. 
Table S-1 Results of gelation test toward common solvents by

cyclo(L- $\beta$-3,7-dimethyloctylasparaginyl-L-phenylalanyl) (1) at $25^{\circ} \mathrm{C}$

\begin{tabular}{lc}
\hline Solvent & MGC $\left(\mathrm{g} \mathrm{L}^{-1}\right)$ \\
\hline Methanol & 44 \\
Ethanol & 28 \\
Acetone & 28 \\
Ethyl acetate & 23 \\
Tetrachloromethane & viscous solution \\
Cyclohexane & viscous solution \\
Benzene & 10 \\
Toluene & 3 \\
Chlorobenzene & 11 \\
Tricaprylin & 15 \\
Trioelein & 15 \\
Soybean oil & 13 \\
Decamethylcyclopentasiloxane & 5 \\
\hline
\end{tabular}

